\title{
AN EXTENSION OF BROUWER'S FIXED-POINT THEOREM TO NONACYCLIC, SET VALUED FUNCTIONS
}

\author{
ROBERT CONNELLY ${ }^{1}$
}

\begin{abstract}
If $f$ is a set valued function defined on an $n$-ball such that each $f(x)$ is a subset of the $n$-ball, and the graph of $f$ is closed, then all that is needed to insure that there is a fixed point $(x \in f(x))$ is that the singularity sets not be too high dimensional. I.e., the dimension of $\left\{x \in B^{n} \mid \tilde{H}^{a}(f(x)) \neq 0\right\}$ is $\leqq n-q-2$. Examples are given to show that the dimension requirements are the best possible. The proof involves defining an analogue of the retraction in the "no retraction" proofs of the Brouwer theorem, and then applying the Leray spectral sequence to the projection of the graph of this retraction onto the $n$-ball.
\end{abstract}

The following is inspired by some recent results of D. G. Bourgin, who has obtained similar results by somewhat different techniques.

We wish to prove an extension of the Brouwer fixed-point theorem in which the function involved is set valued, and each $f(x)$ need not always by acyclic.

Let $X$ and $Y$ be topological spaces, and let $2^{Y}$ denote the set of all subsets of $Y$. We say a function $f: X \rightarrow 2^{Y}$ is upper semicontinuous iff

(a) $\varnothing \neq f(x)$ is closed in $Y$ for all $x \in X$, and

(b) if $F \subset Y$ is closed, then $f^{-1}(F)=\{x \in X \mid f(x) \cap F \neq \varnothing\}$ is closed in $X$.

It is easy to check that, if $Y$ is compact, Hausdorff, then $f$ is upper semicontinuous if and only if the graph of $f$, which is equal to

is closed in $X \times Y$.

$$
\Gamma(f)=\{(x, y) \in X \times Y \mid y \in f(x)\},
$$

We make some standard definitions:

$$
\begin{gathered}
R^{n}=\text { euclidean } n \text {-space and }|| \text { is the standard norm. } \\
B^{n}=\left\{x \in R^{n}|| x \mid \leqq 1\right\}, \quad B^{n}=\left\{x \in R^{n}|| x \mid<1\right\}, \\
S^{n-1}=\partial B^{n}=\left\{x \in R^{n}|| x \mid=1\right\} .
\end{gathered}
$$

Received by the editors June 12, 1972 and, in revised form, May 22, 1973.

AMS (MOS) subject classifications (1970). Primary 55C20, 54H25; Secondary 55B30, 55C10, 55F20, 55H 99 .

Key words and phrases. Brouwer fixed-point theorem, sheaf cohomology, sheaf, Leray spectral sequence, upper semicontinuous, set valued function, dimension, multivalued function.

${ }^{1}$ This research was supported by National Science Foundation grant GP-33960X. 
cl will denote the closure operation (in $B^{n}$ ). In the following, the cohomology theory will be sheaf theoretic cohomology theory, and if coefficients are not mentioned, it will be understood that the coefficient sheaf will be the constant sheaf with each stalk isomorphic to some fixed, nonzero $L$ module, $G$. (In this case the cohomology will coincide with, say, Alexander-Spanier or Čech cohomology in our applications.) See Bredon [1] or Godement [3] for definitions. $\operatorname{dim}_{L}$ will denote the cohomological dimension over $L$ (see Bredon [1, p. 73] for a definition), and in our case is always less than or equal to the covering dimension (Godement [3, p. 236]). $\tilde{H}^{*}$ denotes reduced cohomology.

THEOREM. Let $f: B^{n} \rightarrow 2^{B^{n}}$ be an upper semicontinuous function. Suppose, for $q=0,1, \cdots, n-1$,

$$
\operatorname{dim}_{L} \operatorname{cl}\left\{x \in \stackrel{B}{ }^{n} \mid \tilde{H}^{q}(f(x)) \neq 0\right\} \leqq n-q-2 .
$$

Then there is an $x \in B^{n}$ such that $x \in f(x)$.

If (1) were replaced by the stronger condition that each $f(x)$ be acyclic, then the theorem would be a consequence of a theorem of Eilenberg and Montgomery [2]. The proof below, however, is quite independent of their results. See also O'Neill [4] and [5].

It is interesting to note that, if the dimension requirement is reduced by 1 , then there need not exist a fixed point $\left(x \in B^{n}\right.$ such that $\left.x \in f(x)\right)$. E.g., if we regard $R^{k} \subset R^{n}, k<n$, in the standard way $\left(\left(x_{1}, \cdots, x_{k}\right) \rightarrow\right.$ $\left.\left(x_{1}, \cdots, x_{k}, 0, \cdots, 0\right)\right)$ and thus $B^{k} \subset B^{n}$, we define $g: B^{n} \rightarrow 2^{B^{n}}$ as follows: Let $\pi: B^{n} \rightarrow B^{k}$ be projection onto the first $k$ coordinates. If $x \in B^{n}-B^{k}$, $g(x)=$ the unique point in $\partial B^{n}$ on the ray from $\pi(x)$ through $x$. If $x \in B^{k}$, $g(x)=\pi^{-1}(x) \cap \partial B^{n}$. Define $f(x)=-g(x)$. Clearly $f: B^{n} \rightarrow 2^{B^{n}}$ is upper semicontinuous and has no fixed points. $\left\{x \in \tilde{B}^{n} \mid \tilde{H}^{q}(f(x)) \neq 0\right\}=\varnothing$ except when $q=n-k-1$, and

$$
\begin{aligned}
\operatorname{dim}_{L} \operatorname{cl}\left\{x \in \stackrel{\circ}{ }^{n} \mid \tilde{H}^{n-k-1}(f(x))\right. & \neq 0\} \\
& =\operatorname{dim}_{L} B^{k}=k>n-(n-k-1)-2=k-1 .
\end{aligned}
$$

Proof of Theorem. Suppose $x \notin f(x)$ for all $x \in B^{n}$. We wish to arrive at a contradiction. $\Gamma(f)$ is closed and thus compact and $\Gamma(f) \subset$ $B^{n} \times B^{n}-\Delta=\left\{(x, y) \in B^{n} \times B^{n} \mid x \neq y\right\}$. We wish to find a new set valued function $g: B^{n} \rightarrow 2^{R^{n}-B^{n}}$ such that

$$
\begin{gathered}
\Gamma(g) \text { is also compact, } \\
g(x)=\{x\}, \text { if } x \in \partial B^{n},
\end{gathered}
$$

$$
g(x) \text { is homeomorphic to } f(x) \text {, if } x \in \ddot{B}^{n} \text {. }
$$


We define $g$ as follows: Let $r: B^{n} \times B^{n}-\Delta \rightarrow \partial B^{n}=S^{n-1}$ be defined by $r(x, y)=$ the unique point on $\partial B^{n}$ on the ray from $y$ through $x$. This is the same function as used in the "no retraction" proofs of the Brouwer theorem, and is easily shown to be continuous. Note $r(x, y)=x$, if $x \in \partial B^{n}$. Define a continuous map

by

$$
F: B^{n} \times B^{n}-\Delta \rightarrow R^{n}-\stackrel{\circ}{B}^{n}
$$

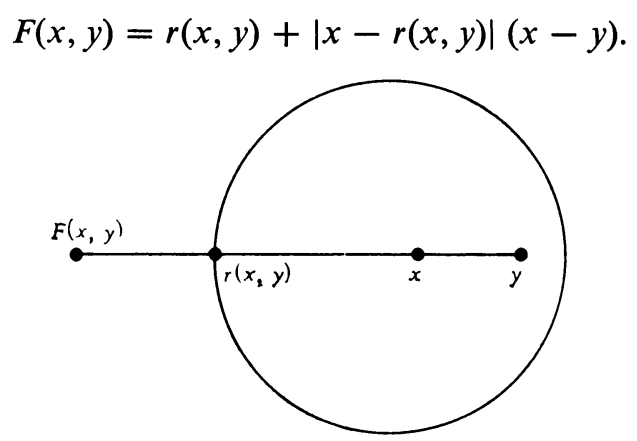

Note if $F(x, y)=F\left(x, y^{\prime}\right)$, then $y=y^{\prime}$, unless $x \in \partial B^{n}$, in which case $F(x, y)=x$. Now define

$$
g(x)=F(x, f(x))=\{F(x, y) \mid y \in f(x)\} .
$$

It is easy to check $g$ satisfies (4) and (5). To show (3) consider the continuous map $\hat{F}: B^{n} \times B^{n}-\Delta \rightarrow B^{n} \times\left(R^{n}-B^{n}\right)$ defined by $\hat{F}(x, y)=$ $(x, F(x, y))$. Then $\hat{F}(\Gamma(f))=\Gamma(g)$ is compact.

Thus, by (4) and (5), for each $q$,

$$
\left\{x \in \stackrel{\circ}{B}^{n} \mid \tilde{H}^{\alpha}(f(x)) \neq 0\right\}=\left\{x \in B^{n} \mid \tilde{H}^{a}(g(x)) \neq 0\right\} .
$$

Let $p_{1}: \Gamma(g) \rightarrow B^{n}$ be projection onto the first factor. We wish to show $\tilde{H}^{n-1}(\Gamma(g))=0$. Note $p_{1}^{-1}(x)$ is homeomorphic to $g(x)$, and $p_{1}$ is a closed map. We now apply the Leray spectral sequence to $p_{1}$ (see Theorem 6.1, p. 140 of [1]). We obtain a spectral sequence with $E_{2}^{p, q}=H^{p}\left(B^{n} ; \mathscr{H}^{q}\left(p_{1}\right)\right)$ converging to $H^{p+q}(\Gamma(g))$, where $\mathscr{H}^{q}\left(p_{1}\right)$ is the sheaf generated by the presheaf $\mathscr{U} \rightarrow H^{q}\left(p_{1}^{-1}(\mathscr{U})\right)$. Since $p_{1}^{-1}(x) \cong g(x)$ is taut (see $[1$, p. 52]) we obtain an isomorphism

$$
\mathscr{H}^{a}\left(p_{1}\right)_{x} \underset{\rightarrow}{\rightrightarrows} H^{q}\left(p_{1}^{-1}(x)\right) \approx H^{a}(g(x)) .
$$

Thus for $q>0$ the stalks, $\mathscr{H}^{q}\left(p_{1}\right)_{x}$, are zero, except possibly on a closed set, $A_{q}=\operatorname{cl}\left\{x \in B^{n} \mid \tilde{H}^{q}(g(x)) \neq 0\right\}$, whose dimension is $\leqq n-q-2$. Since $A_{q}$ is closed we know (by 10.1, p. 51 of [1] or 4.9.1 of [3]), for $q>0$,

$$
E_{2}^{n-1-q, a}=H^{n-q-1}\left(B^{n} ; \mathscr{H}^{q}\left(p_{1}\right)\right) \approx H^{n-q-1}\left(A_{q} ; \mathscr{H}^{q}\left(p_{1}\right) \mid A_{q}\right)=0 .
$$


We have only $E_{2}^{n-1,0}$ left to calculate. Let $G$ denote the constant presheaf on $B^{n}$. Then the following exact sequence regarded as an exact sequence of presheaves,

$$
0 \rightarrow G \rightarrow H^{0}\left(p_{1}^{-1}(\mathscr{U})\right) \rightarrow \tilde{H}^{0}\left(p_{1}^{-1}(\mathscr{U})\right) \rightarrow 0,
$$

induces an exact sequence of sheaves and thus a long exact sequence in cohomology. Let $\tilde{\mathscr{H}}^{0}\left(p_{1}\right)$ denote the sheaf generated by the presheaf defined by $\mathscr{U} \rightarrow \tilde{H}^{0}\left(p_{1}^{-1}(\mathscr{U})\right)$. Then

$$
\rightarrow H^{n-1}\left(B^{n}\right) \rightarrow H^{n-1}\left(B^{n} ; \mathscr{H}^{0}\left(p_{1}\right)\right) \rightarrow H^{n-1}\left(B^{n} ; \tilde{\mathscr{H}}^{0}\left(p_{1}\right)\right)
$$

is exact, and, as before, the stalks, $\tilde{\mathscr{H}}^{0}\left(p_{1}\right)_{x}$, are zero except possibly on a closed set, $A_{0}$, of dimension $\leqq n-2$. Thus, as before,

$$
E_{2}^{n-1,0}=H^{n-1}\left(B^{n} ; \mathscr{H}^{0}\left(p_{1}\right)\right) \approx H^{n-1}\left(B^{n}\right) .
$$

Thus $\tilde{H}^{n-1}(\Gamma(g))=0$.

Lastly we apply the functor, $\tilde{H}^{n-1}$, to the following commutative diagram:

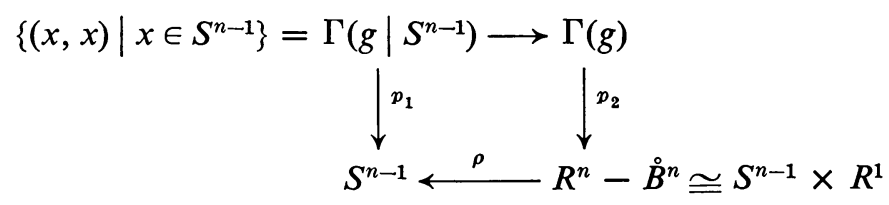

where $p_{2}$ is projection onto the second factor, and $\rho$ is a retraction. We then obtain that $p_{2}^{*} \rho^{*}: \tilde{H}^{n-1}\left(S^{n-1}\right) \rightarrow \tilde{H}^{n-1}(\Gamma(g))$ is one to one, and we finally get a contradiction.

REMARK. Just as with Theorem 6 in [2] we can strengthen the theorem to allow $f: B^{n} \rightarrow 2^{R^{n}}$, but insist that $f\left(S^{n-1}\right) \subset B^{n}$. The proof is as before except that we extend the domains of $r$ and $F$ to the appropriate spaces $\left(B^{n} \times R^{n}-\left\{(x, y) \mid x \neq y,(x, y) \in S^{n-1} \times\left(R^{n}-B^{n}\right)\right\}, r(x, y)=\right.$ last point on $B^{n}$ on the ray through $x$ from $y$ ).

ADDENDUM. The referee has pointed out that a strengthening of the theorem is possible. Namely, in (1) we need only assume that the covering dimension of every subset of $\left\{x \in B^{n} \mid \tilde{H}^{q}(f(x)) \neq 0\right\}$ which is closed in $B^{n}$ is $\leqq n-q-2$. Under this hypothesis the fact that $\tilde{H}^{n-1}(\Gamma(g))=0$ follows directly from Theorem 1 of [6]. In fact the idea above is much the same as in [6], except that Sklyarenko uses the following fact: Let $X$ be paracompact and $M$ be a subset of $X$ such that every subset of $M$ which is closed in $X$ has covering dimension $\leqq d$. (This is called the relative dimension of $M$ in $X$.) Then if $\mathscr{A}$ is any sheaf supported on $M$, $H^{p}(X ; \mathscr{A})=0$ for all $p>d$. The proof of this appears in [7]. 
The author would like to thank R. Livesay and I. Berstein for many helpful conversations and comments.

\section{REFERENCES}

1. G. E. Bredon, Sheaf theory, McGraw-Hill, New York, 1967. MR 36 \#4552.

2. S. Eilenberg and D. Montgomery, Fixed point theorems for multi-valued transformations, Amer. J. Math. 68 (1946), 214-222. MR 8, 51.

3. R. Godement, Topologie algébrique et théorie des faisceaux, Actualités Sci. Indust., no. $1252=$ Publ. Math. Univ. Strasbourg, no. 13, Hermann, Paris, 1958. MR 21 \#1583.

4. B. O'Neill, Fixed points of multi-valued functions, Duke Math. J. 24 (1957), 61-62. MR 18, 752.

5. — Induced homology homomorphisms for set valued maps, Pacific J. Math. 7 (1957), 1179-1184. MR 21 \#2983.

6. E. G. Skljarenko, Some applications of the theory of sheaves in general topology, Uspehi Mat. Nauk 19 (1964), no. 6 (120), 47-70=Russian Math. Surveys 19 (1964), no. 6, 41-62. MR 30 \#1490.

7. - A theorem on mappings which lower the dimension, Bull. Acad. Polon. Sci. Sér. Sci. Math. Astronom. Phys. 10 (1962), 429-432. (Russian) MR 26 \#6933.

Department of Mathematics, Cornell University, Ithaca, New York 14850 